\title{
ANSIEDADE E DEPRESSÃO ENTRE PROFISSIONAIS DE PROGRAMAS DE APRIMORAMENTO PROFISSIONAL
}

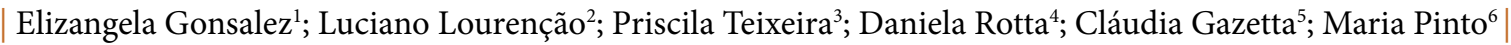

\section{RESUMO}

INTRODUÇÃO: Os programas de formação em serviço são estressantes e podem desencadear o desenvolvimento de ansiedade e depressão, comprometendo as condições de saúde dos profissionais e a qualidade do atendimento prestado aos clientes dos serviços de saúde.

OBJETIVO: Avaliar os níveis de ansiedade e depressão dos profissionais matriculados nos programas de aprimoramento e aperfeiçoamento de uma instituição pública do interior do Estado de São Paulo.

MÉTODOS: Estudo transversal entre 82 profissionais matriculados em programas de formação em serviço de uma instituição pública no Estado de São Paulo, utilizando-se três instrumentos: um para dados socioeconômicos e demográficos, e as Escalas de Ansiedade e Depressão de Beck (Cunha, 2001).

RESULTADOS: Os profissionais apresentam perfil de susceptibilidade aos desgastes físicos e emocionais relacionados ao trabalho - faixa etária de 20 a 25 anos $(47,6)$, sexo feminino $(85,4 \%)$, solteiras $(90,2 \%) .46,8 \%$ dos profissionais apresentaram algum grau de ansiedade e/ou depressão.

CONCLUSÃO: Os níveis de ansiedade e depressão encontrados são significativos e evidenciam a presença de fatores desestimulantes e/ou desgastantes relacionados com a formação profissional nos programas avaliados.

\section{PALAVRAS-CHAVE: Ansiedade; Depressão; Educação em saúde; Internato e residência}

\section{RESUMEN}

"Ansiedad y depresión entre los profesionales de programas de mejoramiento professional"

INTRODUCCIÓN: Programas de formación son estresantes y pueden desencadenar el desarrollo de ansiedad y depresión, comprometer la salud de profesionales y la calidad del servicio prestado a los clientes de los servicios de salud.

OBJETIVO: Evaluar los niveles de ansiedad y depresión de profesionales matriculados en los programas de mejora y mejora de una institución pública en el Estado de São Paulo en Brasil.

METODOLOGÍA: Estudio transversal entre los 82 profesionales inscritos en los programas de formación en servicio de una institución pública en el Estado de Sao Paulo, utilizando tres instrumentos: una para los datos socioeconómicos y demográficos, y las Escalas de Ansiedad y Depresión de Beck (Cunha, 2001).

RESULTADOS: Los profesionales muestran perfil de susceptibilidad a un desgaste emocional y física relacionada con el trabajo - grupo de edad de $20-25$ años $(47,6)$, mujeres $(85,4 \%)$, sola $(90,2 \%) .46,8 \%$ de los encuestados tenía algún grado de ansiedad y / o depresión.

CONCLUSIÓN: Los niveles de ansiedad y depresión que se encuentran son significativos y muestran la presencia de factores desalentadores y/o estresante relacionados con la formación profesional en los programas evaluados.

\section{DESCRIPTORES: Ansiedad; Depresión; Educación em Salud;} Internado y Residencia

\begin{abstract}
"Anxiety and depression between workers at professional improvement programs"

INTRODUCTION: In-service training programs are stressful and can trigger the development of anxiety and depression, compromising the health conditions of professionals and the quality of care provided to users of health services.

AIM: To evaluate the anxiety and depression levels of professionals enrolled in the improvement and improvement programs of a public institution in the interior of the State of São Paulo.

METHODS: Cross-sectional study of 82 professionals enrolled in inservice training programs of a public institution in the State of São Paulo, using three instruments: one for socioeconomic and demographic data, the Beck Anxiety and Beck Depression Scales (Cunha, 2001).

RESULTS: Professionals presented a susceptibility profile to physical and emotional exhaustion related to work - age range of 20 to 25 years (47.6), female (85.4\%), single (90.2\%). $46.8 \%$ of the professionals presented some degree of anxiety and / or depression.

CONCLUSION: The levels of anxiety and depression found are significant and show the presence of discouraging and / or debilitating factors related to professional training programs evaluated.
\end{abstract}

KEYWORDS: Anxiety; Depression; Health education; Internship and residency

Submetido em 20-09-2017

Aceite em 17-11-2017

1 Enfermeira; Mestre em Enfermagem, gianinicaberlin@hotmail.com

2 Enfermeiro; Doutor em Ciências da Saúde; Professor Titular-Livre na Universidade Federal do Rio Grande, 90040-060, Rio Grande, Brasil, luciano.famerp@gmail.com

3 Psicóloga; Mestre em Enfermagem, priscilapsicologa@yahoo.com.br

4 Enfermeira; Mestre em Enfermagem, danielarotta@yahoo.com.br

5 Enfermeira; Doutora em Enfermagem; Professor Adjunta na Faculdade de Medicina de São José do Rio Preto, Departamento de Enfermagem em Saúde Coletiva e Orientação Profissional, 15090-000 São José do Rio Preto, SP, Brasil, claudiagazetta@yahoo.com.br

6 Enfermeira; Doutora em Enfermagem; Professora Adjunta na Faculdade de Medicina de São José do Rio Preto, Departamento de Enfermagem Geral, mariahelena@famerp.br

Citação: Gonsalez, E. G., Lourenção, L. G., Teixeira, P. R., Rotta, D. S., Gazetta, C. E., \& Pinto, M. H. (2017). Ansiedade e depressão entre profissionais de programas de aprimoramento profissional. Revista Portuguesa de Enfermagem de Saúde Mental (18), 51-58. doi: 10.19131/rpesm.0192 


\section{INTRODUÇÃO}

Os programas de aprimoramento e aperfeiçoamento profissional, desenvolvidos na modalidade de formação em serviço, sob supervisão, objetivam transmitir e desenvolver nos profissionais de saúde recém-graduados, os conhecimentos inerentes às ciências da saúde, de forma a atender as demandas sociais e clínico-epidemiológicas, contribuindo para a melhoria do atendimento prestado à comunidade usuária do Sistema Único de Saúde (SUS) (Lourenção, Moscardini \& Soler, 2013; Secretaria de Estado da Saúde, 2015). Possibilitam, portanto, que estes profissionais adquiram conhecimentos específicos e habilidades nas respectivas áreas de conhecimento. No Estado de São Paulo, o Programa de Aprimoramento Profissional da Secretaria de Estado da Saúde (PAP/SES), oferece oportunidades de formação em diversas áreas da saúde - exceto medicina, por meio de instituições vinculadas ao SUS (Secretaria de Estado da Saúde, 2015).

A Faculdade de Medicina de São José do Rio Preto (FAMERP), instituição de ensino pública do Estado de São Paulo, além das vagas para o Programa de Aprimoramento Profissional da Secretaria de Estado da Saúde, possui um Programa de Aperfeiçoamento Profissional, desenvolvido nos mesmos moldes do Programa de Aprimoramento Profissional da Secretaria de Estado da Saúde e mantido com recursos próprios, com vagas em diferentes especialidades, de diferentes áreas da saúde (Faculdade de Medicina de São José do Rio Preto [FAMERP], 2015). Estes programas têm como objetivo capacitar os profissionais para exercerem a profissão com maior qualidade, atendendo a demanda dos clientes do sistema público de saúde (Secretaria de Estado da Saúde, 2015).

Os programas de formação em serviço são considerados estressantes, podendo desencadear o desenvolvimento de ansiedade e depressão, comprometendo as condições de saúde, a qualidade de vida dos profissionais e a qualidade do atendimento prestado aos usuários dos serviços de saúde (Rotta et al., 2016; Carvalho, Melo-Filho, Carvalho \& Amorim, 2013; Lourenção et al., 2013).

Fatores como a presença de pacientes e doenças graves, a convivência com o sofrimento e a morte, a pressão para tomada de decisões, o medo de errar, o excesso de trabalho e, muitas vezes, a falta de infraestrutura adequada dos serviços de saúde, causam temores entre os profissionais, desestruturando o seu desempenho e gerando desgastes físicos e emocionais, como ansiedade e depressão (Carvalho et al., 2013; Lourenção et al., 2013).
A ansiedade pode ser definida como uma preparação do organismo para agir diante de estímulos e contingências que ameaçam a integridade da sua estrutura. É um sentimento vago, indescritível e desagradável, de medo e apreensão, caracterizado por tensão ou desconforto, derivado de antecipação do perigo, de algo estranho, desconhecido. Quando a ansiedade extrapola os limites da normalidade, surgem os transtornos de ansiedade, considerados muito comuns, mas que causam sofrimento e comprometimento funcional importante, podendo impedir o adequado funcionamento do profissional (Gomes \& Oliveira, 2013; Schmidt, Dantas \& Marziale, 2011).

Os sinais e sintomas de ansiedade podem ser caracterizados como sensações físicas de aceleração respiratória, alteração do batimento cardíaco, polaquiúria, diarréia, falta de forças nas pernas, palidez, contrações ou relaxamento dos músculos faciais, sudorese, tremores, etc. (Gomes \& Oliveira, 2013; Apóstolo, Figueiredo, Mendes \& Rodrigues, 2011; Schmidt et al., 2011).

A depressão é considerada uma das principais causas de incapacidade em todo o mundo. Estima-se que 350 milhões de pessoas são afetadas pela depressão, sendo mais comum entre mulheres. É caracterizada por um emaranhado de sensações e alterações no comportamento dos indivíduos, como tristeza, perda de interesse ou prazer, sentimentos de culpa ou baixa auto-estima, distúrbios do sono ou do apetite, cansaço e falta de concentração (World Health Organization [WHO], 2015).

A depressão pode ser de longa duração ou recorrente, prejudicando a capacidade das pessoas no trabalho e na vida diária. Entre as alterações que podem ocorrer e estarem relacionadas com um quadro depressivo destacam-se: afastamento das atividades sociais, perda de interesse nas atividades profissionais, acadêmicas e lúdicas, perda do prazer nas relações interpessoais, sentimento de culpa ou auto-depreciação, baixa autoestima, desesperança, apetite e sono alterados, sensação de falta de energia e dificuldade de concentração.

Além das manifestações nos comportamentos dos indivíduos e nas suas formas de se comportar e se relacionar, podem ser identificadas algumas alterações em aspectos físicos, além de surgirem cefaleias constantes, disfunções relacionadas com o sono, náuseas, dores na região das costas, perda ou diminuição do interesse sexual, entre outras. Diferentemente da tristeza e do luto, a depressão não é uma sensação voluntária do indivíduo, mas algo indesejável (WHO, 2015; Apóstolo, et al., 2011). 
Em virtude dos desgastes físicos e emocionais vivenciados pelos profissionais durante o processo de formação nos programas de aprimoramento e aperfeiçoamento profissional, capazes de desestruturar o desempenho laboral durante o treinamento, torna-se importante verificar o nível de ansiedade e depressão destes indivíduos, buscando a humanização da vida profissional e pessoal, a partir da promoção da saúde física e mental destes profissionais.

Assim, este estudo objetivou avaliar os níveis de ansiedade e depressão dos profissionais matriculados nos programas de aprimoramento e aperfeiçoamento de uma instituição pública do interior do Estado de São Paulo.

\section{MÉTODOS}

Estudo transversal entre os profissionais matriculados nos programas de aprimoramento e aperfeiçoamento da Faculdade de Medicina de São José do Rio Preto, no ano de 2013.

Ambos são programas de pós-graduação multiprofissionais, que objetivam a formação profissional em serviço, sob supervisão, desenvolvidos com jornada semanal de 40 (quarenta) horas, constituindo-se de atividades teóricas $(20 \%)$ e práticas $(80 \%)$, conforme determina a legislação (Faculdade de Medicina de São José do Rio Preto, 2015).

A população do estudo foi composta por todos profissionais matriculados nos programas de aprimoramento e aperfeiçoamento da Faculdade de Medicina de São José do Rio Preto, que consentiram participar, depois de informados sobre os objetivos e a finalidade do estudo, totalizando 82 profissionais de diferentes áreas, dos quais 31 eram aprimorandos e 51 aperfeiçoandos. Todos frequentando o semestre letivo entre 2013 e 2014. O critério de inclusão no estudo foi ser aprimorando ou aperfeiçoando matriculado no programa. Foram excluídos aqueles que estavam afastados das atividades profissionais por qualquer natureza durante o período de coleta dos dados.

Para a coleta de dados foram utilizados três instrumentos autoaplicáveis: um instrumento elaborado pelos autores, contendo dados pessoais como idade, sexo, estado civil, categoria profissional, renda familiar, se está satisfeito com o programa e se já pensou em desistir do programa (dados demográficos e socioeconômicos); a Escala de Ansiedade de Beck ou Inventário de Ansiedade de Beck (BAI); e a Escala de Depressão de Beck ou Inventário de Depressão de Beck (BDI-II).
O BAI foi desenvolvido por Beck, Epstein, Brown e Steer, em 1988 e adaptado para o Brasil por Cunha (2001), apresentando bons coeficientes de fidedignidade e validade. É composto por 21 questões que abordam diversos itens relacionados aos sintomas depressivos como desesperança, irritabilidade e cognições como culpa ou sentimentos de estar sendo punida, assim como sintomas físicos como fadiga, perda de peso e diminuição da libido. A aplicação do BAI seguiu as recomendações do Manual de Escalas Beck. Após leitura das instruções aos participantes do estudo, foi entregue o protocolo do teste para respostas. A correção foi realizada por uma psicóloga e a classificação dos níveis de ansiedade dos profissionais seguiu a seguinte classificação: 0 a 10 pontos = Nível Mínimo de Ansiedade; 11 a 19 pontos = Nível Leve de Ansiedade; 20 a 30 pontos = Nível Moderado de Ansiedade; 31 a 63 pontos = Nível Grave de Ansiedade (Cunha, 2001).

O BDI-II, criado por Beck, Ward, Mendelson, Mock e Erbaugh em 1961, apresenta coeficientes de fidedignidade e evidências de validade satisfatórias para o Brasil (Cunha, 2001). É composto por 21 questões que abordam diversos itens relacionados aos sintomas depressivos como desesperança, irritabilidade e cognições como culpa ou sentimentos de estar sendo punida, assim como sintomas físicos como fadiga, perda de peso e diminuição da libido. Como no BAI, a aplicação do BDI-II seguiu as recomendações do Manual de Escalas Beck. Após leitura das instruções aos participantes do estudo, foi entregue o protocolo do teste para respostas. A correção foi realizada por uma psicóloga e a classificação dos níveis de depressão dos profissionais seguiu a seguinte classificação: 0 a 11 pontos = Nível Mínimo de Depressão; 12 a 19 pontos = Nível Leve de Depressão; 20 a 35 pontos $=$ Nível Moderado de Depressão; 36 a 63 pontos = Nível Grave de Depressão (Cunha, 2001).

Todos os profissionais matriculados nos programas, que não estavam afastados no momento da coleta dos dados, participaram da pesquisa e responderam ao questionário no início de uma aula teórica do programa, com a prévia permissão do professor responsável.

A análise dos dados foi realizada com o programa Statistical Package for Social Sciences (SPSS), versão 17.0. Os dados demográficos e socioeconômicos foram utilizados para caracterizar a população do estudo. Os níveis de ansiedade de depressão foram categorizados em ausência de depressão/ansiedade, depressão/ansiedade leve, depressão/ansiedade moderada e depressão/ ansiedade grave e apresentadas segundo as variáveis sociodemográficas dos profissionais. 
Para testar associações entre as variáveis sociodemográficas e os níveis de ansiedade e depressão utilizou-se o teste qui-quadrado, com nível de significância de 5\%. O teste de concordância de Kappa foi utilizado para medir a relação entre os níveis de ansiedade e depressão, considerando significância de $5 \%$.

Respeitando os preceitos Éticos de Pesquisas envolvendo seres humanos, este projeto foi submetido à apreciação do Comitê de Ética em Pesquisa da Faculdade de Medicina de São José do Rio Preto, sob Protocolo CAAE 17116413.4.0000.5415 e aprovado em 11 de junho de 2013 com o Parecer n. 319.203.

\section{RESULTADOS}

Participaram do estudo 82 profissionais de diferentes categorias, dos quais 31 eram aprimorandos e 51 aperfeiçoandos. Em relação às categorias profissionais, 21 $(25,6 \%)$ eram enfermeiros, dois $(2,4 \%)$ farmacêuticos, $25(30,5 \%)$ fisioterapeutas, seis $(7,3 \%)$ fonoaudiólogos, duas $(2,4 \%)$ nutricionistas, 12 (14,6\%) psicólogos, oito $(9,8 \%)$ assistentes sociais e seis $(7,3)$ terapeutas ocupacionais.

Observou-se prevalência do sexo feminino (70 - 85,4\%). A faixa etária variou de 22 a 32 anos, com idade mediana de 25 anos, sendo que 39 (47,6\%) profissionais tinham entre 20 e 25 anos, 29 (35,4\%) entre 26 e 30 anos, um $(1,2 \%)$ entre 31 e 35 anos.

Em relação ao estado civil, observou-se que a maioria era solteira $(74-90,2 \%)$. Sete $(8,5 \%)$ profissionais tinham rendimento familiar de até 1 salário mínimo; 45 (54,9\%), de dois a cinco salários mínimos; 23 (28,0\%), de seis a 10 salários mínimos e seis $(7,3 \%)$, mais de 10 salários mínimos. Destaca-se que 49 (59,8\%) profissionais referiram estar satisfeitos com o programa no qual está matriculado, e 50 (61,0\%) já pensaram em desistir do programa.

A análise do nível de ansiedade (Tabela 1) evidenciou que $46,80 \%$ dos profissionais apresentaram algum grau de ansiedade, sendo 37,0\% com ansiedade leve, 4,9\% ansiedade moderada e 4,9\% ansiedade grave. Os maiores percentuais de ansiedade foram observados em profissionais das áreas de: Farmácia - 100\% dos profissionais com ansiedade leve; Serviço Social - 62,5\% dos profissionais com ansiedade leve; Nutrição - 50\% dos profissionais com ansiedade leve e 50\% com ansiedade grave; Fisioterapia: $37,5 \%$ dos profissionais com ansiedade leve e $12,5 \%$ com ansiedade moderada; Enfermagem - 33,3\% dos profissionais com ansiedade leve e $14,3 \%$ com ansiedade grave.
Tabela 1 - Níveis de Ansiedade, Variáveis Sociodemográficas dos Profissionais Matriculados nos Programas de Aprimoramento e Aperfeiçoamento Profissional da Faculdade de Medicina de São José do Rio Preto. São José do Rio Preto, São Paulo, 2013-2014

\begin{tabular}{|c|c|c|c|c|c|}
\hline & \multicolumn{5}{|c|}{ Ansiedade } \\
\hline & $\begin{array}{c}\text { Ausência } \\
\text { n (\%) }\end{array}$ & $\begin{array}{l}\text { Leve } \\
\text { n (\%) }\end{array}$ & $\begin{array}{c}\text { Moderada } \\
\text { n (\%) }\end{array}$ & $\begin{array}{l}\text { Grave } \\
\text { n }(\%)\end{array}$ & Valor-p \\
\hline \multicolumn{6}{|l|}{ Categoria Profissional } \\
\hline $\begin{array}{l}\text { Enfermagem } \\
(\mathrm{n}=21)\end{array}$ & $11(52,4)$ & $7(33,3)$ & - & $3(14,3)$ & \multirow{9}{*}{0,110} \\
\hline Farmácia $(\mathrm{n}=02)$ & - & $2(100,0)$ & - & - & \\
\hline Fisioterapia $(\mathrm{n}=24)$ & $12(50,0)$ & $9(37,5)$ & $3(12,5)$ & - & \\
\hline $\begin{array}{l}\text { Fonoaudiologia } \\
(\mathrm{n}=06)\end{array}$ & $4(66,7)$ & $2(33,3)$ & - & - & \\
\hline Nutrição $(\mathrm{n}=02)$ & - & $1(50,0)$ & - & $1(50,0)$ & \\
\hline Psicologia $(n=12)$ & $8(66,7)$ & $3(25,0)$ & $1(8,3)$ & - & \\
\hline $\begin{array}{l}\text { Serviço Social } \\
(\mathrm{n}=08)\end{array}$ & $3(37,5)$ & $5(62,5)$ & - & - & \\
\hline $\begin{array}{l}\text { Terapia Ocupacio- } \\
\text { nal }(\mathrm{n}=06)\end{array}$ & $5(83,3)$ & $1(16,7)$ & - & - & \\
\hline Total $(\mathrm{n}=81)$ & $43(53,1)$ & $30(37,0)$ & $4(4,9)$ & $4(4,9)$ & \\
\hline \multicolumn{6}{|l|}{ Sexo } \\
\hline Masculino $(\mathrm{n}=12)$ & $9(75,0)$ & $3(25,0)$ & - & - & \multirow{3}{*}{0,359} \\
\hline Feminino $(\mathrm{n}=69)$ & $34(49,3)$ & $27(39,1)$ & $4(5,8)$ & $4(5,8)$ & \\
\hline Total $(\mathrm{n}=81)$ & $43(53,1)$ & $30(37,0)$ & $4(4,9)$ & $4(4,9)$ & \\
\hline \multicolumn{6}{|l|}{ Faixa etária } \\
\hline $\begin{array}{l}20-25 \text { anos } \\
(n=46)\end{array}$ & $23(50,0)$ & $18(39,1)$ & $2(4,3)$ & $3(6,5)$ & \multirow{4}{*}{0,398} \\
\hline $\begin{array}{l}26-30 \text { anos } \\
(n=30)\end{array}$ & $18(60,0)$ & $10(33,3)$ & $1(3,3)$ & $1(3,3)$ & \\
\hline $\begin{array}{l}31-35 \text { anos } \\
(n=03)\end{array}$ & $1(33,3)$ & $1(33,3)$ & $1(33,3)$ & - & \\
\hline Total $(\mathrm{n}=79)$ & $42(53,2)$ & $29(36,7)$ & $4(5,1)$ & $4(5,1)$ & \\
\hline \multicolumn{6}{|l|}{ Estado civil } \\
\hline Casado $(\mathrm{n}=04)$ & $2(50,0)$ & $1(25,0)$ & - & $1(25,0)$ & \multirow{4}{*}{0,231} \\
\hline Solteiro $(\mathrm{n}=73)$ & $40(54,8)$ & $27(37,0)$ & $3(4,1)$ & $3(4,1)$ & \\
\hline Outros $(\mathrm{n}=04)$ & $1(25,0)$ & $2(50,0)$ & $1(25,0)$ & - & \\
\hline Total $(\mathrm{n}=81)$ & $43(53,1)$ & $30(37,0)$ & $4(4,9)$ & $4(4,9)$ & \\
\hline \multicolumn{6}{|c|}{ Renda Familiar (salários mínimos) } \\
\hline Até $1(\mathrm{n}=07)$ & $4(57,1)$ & $2(28,6)$ & - & $1(14,3)$ & \multirow{5}{*}{0,817} \\
\hline 2 a $5(n=45)$ & $23(51,1)$ & $17(37,8)$ & $3(6,7)$ & $2(4,4)$ & \\
\hline 6 a $10(n=22)$ & $12(54,5)$ & $9(40,9)$ & - & $1(4,5)$ & \\
\hline Mais de $10(n=06)$ & $3(50,0)$ & $2(33,3)$ & $1(16,7)$ & - & \\
\hline Total $(\mathrm{n}=80)$ & $42(52,5)$ & $30(37,5)$ & $4(5,0)$ & $4(5,0)$ & \\
\hline \multicolumn{6}{|c|}{ Satisfeito com o programa } \\
\hline $\operatorname{Sim}(n=48)$ & $26(54,2)$ & $18(37,5)$ & $3(6,3)$ & $1(2,1)$ & \multirow{3}{*}{0,496} \\
\hline Não (n = 33) & $17(51,5)$ & $12(37,5)$ & $1(3,0)$ & $3(9,1)$ & \\
\hline Total $(\mathrm{n}=81)$ & $43(53,1)$ & $30(37,0)$ & $4(4,9)$ & $4(4,9)$ & \\
\hline \multicolumn{6}{|c|}{ Pensou em desistir do programa } \\
\hline $\operatorname{Sim}(n=49)$ & $23(46,9)$ & $18(36,7)$ & $4(8,2)$ & $4(8,2)$ & \multirow{3}{*}{0,106} \\
\hline Não (n = 32) & $20(62,5)$ & $12(37,5)$ & - & - & \\
\hline Total $(\mathrm{n}=81)$ & $43(53,1)$ & $30(37,0)$ & $4(4,9)$ & $4(4,9)$ & \\
\hline
\end{tabular}


Entre os 33 profissionais que referiram não estar satisfeitos com o programa, 49,6\% apresentaram ansiedade, sendo 37,5\% com ansiedade leve, 3,0\% moderada e 9,1\% grave. Já entre aqueles que pensaram em desistir do programa $(\mathrm{n}=81), 53,1 \%$ apresentaram ansiedade, dos quais: $36,7 \%$ tiveram ansiedade leve, $8,2 \%$ moderada e $8,2 \%$ ansiedade grave.

Tabela 2 - Níveis de Depressão, Variáveis Sociodemográficas dos Profissionais Matriculados nos Programas de Aprimoramento e Aperfeiçoamento Profissional da Faculdade de Medicina de São José do Rio Preto. São José do Rio Preto, São Paulo, 2013-2014

\begin{tabular}{|c|c|c|c|c|c|}
\hline & \multicolumn{5}{|c|}{ Depressão } \\
\hline & $\begin{array}{l}\text { Ausência } \\
\text { n (\%) }\end{array}$ & $\begin{array}{l}\text { Leve } \\
\text { n (\%) }\end{array}$ & $\begin{array}{l}\text { Modera- } \\
\text { da n (\%) }\end{array}$ & $\begin{array}{l}\text { Grave } \\
\text { n (\%) }\end{array}$ & $\begin{array}{l}\text { Valor- } \\
\mathbf{p}\end{array}$ \\
\hline \multicolumn{6}{|l|}{ Categoria Profissional } \\
\hline Enfermagem $(\mathrm{n}=20)$ & $13(65,0)$ & $6(30,0)$ & $1(5,0)$ & - & \multirow{9}{*}{0,961} \\
\hline Farmácia $(n=02)$ & $1(50,0)$ & $1(50,0)$ & - & - & \\
\hline Fisioterapia $(\mathrm{n}=25)$ & $21(84,0)$ & $3(12,0)$ & $1(4,0)$ & - & \\
\hline Fonoaudiologia $(\mathrm{n}=06)$ & $5(83,3)$ & $1(16,7)$ & - & - & \\
\hline Nutrição (n = 02) & $1(50,0)$ & $1(50,0)$ & - & - & \\
\hline Psicologia $(n=12)$ & $8(66,7)$ & $4(33,3)$ & - & - & \\
\hline Serviço Social $(\mathrm{n}=08)$ & $6(75,0)$ & $2(25,0)$ & - & - & \\
\hline $\begin{array}{l}\text { Terapia Ocupacional } \\
(\mathrm{n}=05)\end{array}$ & $4(80,0)$ & $1(20,0)$ & - & - & \\
\hline Total $(\mathrm{n}=80)$ & $59(73,8)$ & $19(23,8)$ & $2(2,5)$ & - & \\
\hline \multicolumn{6}{|l|}{ Sexo } \\
\hline Masculino $(\mathrm{n}=12)$ & $11(91,7)$ & $1(8,3)$ & - & - & \multirow{3}{*}{0,304} \\
\hline Feminino $(\mathrm{n}=68)$ & $48(70,6)$ & $18(26,5)$ & $2(2,9)$ & - & \\
\hline Total $(\mathrm{n}=80)$ & $59(73,8)$ & $19(23,8)$ & $2(2,5)$ & - & \\
\hline \multicolumn{6}{|l|}{ Faixa etária } \\
\hline $20-25 \operatorname{anos}(n=46)$ & $31(67,4)$ & $13(28,3)$ & $2(4,3)$ & - & \multirow{4}{*}{0,404} \\
\hline $26-30 \operatorname{anos}(n=29)$ & $25(86,2)$ & $4(13,8)$ & - & - & \\
\hline $31-35$ anos $(n=03)$ & $2(66,7)$ & $1(33,3)$ & - & - & \\
\hline Total $(\mathrm{n}=78)$ & $58(74,4)$ & $18(23,1)$ & $2(2,6)$ & - & \\
\hline \multicolumn{6}{|l|}{ Estado civil } \\
\hline Casado $(\mathrm{n}=04)$ & $3(75,0)$ & - & $1(25,0)$ & - & \multirow{4}{*}{0,001} \\
\hline Solteiro $(\mathrm{n}=72)$ & $54(75,0)$ & $18(25,0)$ & - & - & \\
\hline Outros $(\mathrm{n}=04)$ & $2(50,0)$ & $1(25,0)$ & $1(25,0)$ & - & \\
\hline Total $(\mathrm{n}=80)$ & $59(73,8)$ & $19(23,8)$ & $2(2,5)$ & - & \\
\hline \multicolumn{6}{|c|}{ Renda Familiar (salários mínimos) } \\
\hline Até $1(\mathrm{n}=07)$ & $6(85,7)$ & $1(14,3)$ & - & - & \multirow{5}{*}{0,502} \\
\hline 2 a $5(n=43)$ & $28(65,1)$ & $13(30,2)$ & $2(4,7)$ & - & \\
\hline 6 a $10(n=23)$ & $18(78,3)$ & $5(21,7)$ & - & - & \\
\hline Mais de $10(n=06)$ & $6(100,0)$ & - & - & - & \\
\hline Total $(\mathrm{n}=79)$ & $58(73,4)$ & $19(24,1)$ & $2(2,5)$ & - & \\
\hline \multicolumn{6}{|l|}{ Satisfeito com o programa } \\
\hline $\operatorname{Sim}(n=49)$ & $41(83,7)$ & $8(16,3)$ & - & - & \multirow{3}{*}{0,020} \\
\hline Não $(\mathrm{n}=31)$ & $18(58,1)$ & $11(35,5)$ & $2(6,5)$ & - & \\
\hline Total $(\mathrm{n}=80)$ & $59(73,8)$ & $19(23,8)$ & $2(2,5)$ & - & \\
\hline \multicolumn{6}{|c|}{ Pensou em desistir do programa } \\
\hline $\operatorname{Sim}(\mathrm{n}=49)$ & $31(63,3)$ & $16(32,7)$ & $2(4,1)$ & - & \multirow{3}{*}{0,025} \\
\hline Não $(\mathrm{n}=31)$ & $28(90,3)$ & $3(9,7)$ & - & - & \\
\hline Total $(\mathrm{n}=80)$ & $59(73,8)$ & $19(23,8)$ & $2(2,5)$ & - & \\
\hline
\end{tabular}

Não houve diferença estatística entre os níveis de ansiedade e as variáveis sociodemográficas.

A Tabela 2 apresenta os níveis de depressão, segundo as variáveis sociodemográficas dos profissionais estudados. Destaca-se que nenhum profissional apresentou níveis graves de depressão. Contudo, 26,3\% dos profissionais apresentaram depressão leve e/ou moderada, sendo que $5,0 \%$ dos enfermeiros e $4,0 \%$ dos fisioterapeutas apresentaram depressão moderada. Em todas as categorias profissionais foi observada depressão leve, cujos índices foram: 50,0\% dos farmacêuticos e nutricionistas; 33,3\% dos psicólogos; $30,0 \%$ dos enfermeiros; $25,0 \%$ dos assistentes sociais; $20,0 \%$ dos terapeutas ocupacionais; $16,7 \%$ dos fonoaudiólogos e 12,0\% dos fisioterapeutas.

Dos 31 profissionais que referiram não estar satisfeitos com o programa, $36,8 \%$ apresentaram depressão, sendo $35,5 \%$ com depressão leve e 6,5\% moderada. Entre os que pensaram em desistir do programa $(n=49), 32,7 \%$ apresentaram depressão leve e $4,1 \%$, depressão moderada, totalizando $36,8 \%$ dos profissionais com algum nível de depressão.

Observou-se diferença estatística significante entre depressão e estado civil $(\mathrm{p}=0,001)$, depressão e satisfação com o programa $(\mathrm{p}=0,020)$, e depressão e desejo de desistir do programa $(\mathrm{p}=0,025)$, evidenciando que essas variáveis contribuem para o surgimento da depressão.

A Tabela 3 apresenta a relação entre ansiedade e depressão nos profissionais, mostrando que $45,6 \%$ dos profissionais apresentaram sintomas de ansiedade associada ou não à depressão. Ainda, destaca-se que, 20,3\% dos profissionais apresentaram ansiedade e depressão associadas. Houve baixa concordância entre profissionais com ansiedade e depressão $(K=0,171)$ e não houve significância estatística $(\mathrm{p}=0,089)$.

Tabela 3 - Relação entre Ansiedade e Depressão nos

Profissionais Matriculados nos Programas de

Aprimoramento e Aperfeiçoamento Profissional da Faculdade de Medicina de São José do Rio Preto.

São José do Rio Preto, São Paulo, 2013-2014

\begin{tabular}{|c|c|c|c|c|c|c|c|}
\hline \multirow[b]{2}{*}{ Variáveis } & \multicolumn{4}{|c|}{ Depressão } & \multirow[b]{2}{*}{ Total } & \multirow[b]{2}{*}{ Kappa } & \multirow[b]{2}{*}{$\begin{array}{c}\text { p- } \\
\text { valor }\end{array}$} \\
\hline & $\begin{array}{c}\text { Ausência } \\
\text { n (\%) }\end{array}$ & $\begin{array}{l}\text { Leve } \\
\text { n (\%) }\end{array}$ & $\begin{array}{c}\text { Mod- } \\
\text { erada } n \\
\quad(\%)\end{array}$ & $\begin{array}{l}\text { Grave } \\
\text { n (\%) }\end{array}$ & & & \\
\hline \multicolumn{6}{|l|}{ Ansiedade } & \multirow{6}{*}{0,171} & \multirow{6}{*}{0,089} \\
\hline Ausência & $38(65,5)$ & $5(26,3)$ & - & - & $\begin{array}{c}43 \\
(54,4)\end{array}$ & & \\
\hline Leve & $19(32,8)$ & $8(42,1)$ & $1(50,0)$ & - & $\begin{array}{c}28 \\
(35,4)\end{array}$ & & \\
\hline Moderada & $1(1,7)$ & $3(15,8)$ & - & - & $4(5,1)$ & & \\
\hline Grave & - & $3(15,8)$ & $1(50,0)$ & - & $4(5,1)$ & & \\
\hline Total & $58(73,4)$ & $\begin{array}{c}19 \\
(24,0)\end{array}$ & $2(2,6)$ & - & $\begin{array}{c}79 \\
(100,0)\end{array}$ & & \\
\hline
\end{tabular}




\section{DISCUSSÃO}

A distribuição dos profissionais observada neste estudo está relacionada ao perfil institucional dos programas estudados. Ligado à Faculdade de Medicina de São José do Rio Preto, o Hospital de Base está entre os maiores complexos hospitalares do Estado de São Paulo. É referência para o atendimento de mais de dois milhões de habitantes dos 102 municípios que integram a Direção Regional de Saúde de Rio Preto (DRS 15) e conta com equipes multidisciplinares formadas por médicos, enfermeiros, nutricionistas, terapeutas ocupacionais, assistentes sociais, fisioterapeutas, psicólogos e cirurgiões-dentistas, entre outros profissionais (Hospital de Base de São José do Rio Preto, 2016).

Este perfil é coerente com o público alvo dos programas multidisciplinares de Aprimoramento Profissional da Secretaria de Estado da Saúde de São Paulo e Aperfeiçoamento Profissional da Faculdade de Medicina de São José do Rio Preto, que ofertam 113 vagas, sendo 55 para o aprimoramento e 58 vagas para o aperfeiçoamento (FAMERP, 2015; Secretaria de Estado da Saúde, 2015).

O perfil sociodemográfico dos profissionais deste estudo está em concordância com o perfil identificado em outros estudos, ou seja, mulheres, jovens, solteiras, com rendimento familiar médio entre dois e cinco salários mínimos (Fernandes, Miyazaki \& Silvares, 2015; Lourenção et al., 2013; Franco, Barros, Nogueira-Martins \& Zeitoun, 2011).

De acordo com a literatura, profissionais com este perfil (mulheres, jovens, solteiras e no início da carreira) estão mais susceptíveis a desgastes físicos e emocionais relacionados ao trabalho (Franco et al., 2011).

Este estudo observou que um elevado percentual dos profissionais, por algum motivo não já pensou em desistir do programa de formação.

Estudo sobre qualidade de vida, realizado em 2009 com profissionais desses mesmos programas, identificou comprometimento da vida sexual e das atividades da vida cotidiana dos profissionais, além de dificuldades de enfrentamento das situações estressantes (Lourenção et al., 2013), evidenciando a existência de fatores desestimulantes e/ou desgastantes relacionados ao processo de formação.

Esses resultados também são semelhantes aos encontrados em outro estudo realizado numa universidade pública da região sul do Brasil, com residentes multiprofissionais (Guido, Silva, Goulart, Bolzan \& Lopes, 2012).
$\mathrm{Na}$ avaliação da ansiedade, observou-se que 46,80\% dos profissionais apresentaram algum grau de ansiedade, corroborando com os resultados encontrados por outros estudos com residentes multiprofissionais e residentes médicos, nos quais os percentuais de profissionais com ansiedade variou entre 40 e 60\% (Carvalho et al., 2013; Guido et al., 2012; Silva, Koch, Sousa, Gasparetto \& Buys, 2010).

Apesar dos resultados não mostrarem diferença estatisticamente significante no percentual de profissionais com ansiedade em relação às variáveis sociodemográficas analisadas, algumas questões chamam a atenção, como o percentual de profissionais de Farmácia, Serviço Social e Nutrição ansiosos; o percentual de mulheres com ansiedade foi superior ao dos homens; houve maior percentual de ansiosos entre os profissionais mais jovens; quase a metade dos profissionais insatisfeitos apresentava ansiedade e mais de $50,0 \%$ dos profissionais que pensaram em desistir do programa apresentavam ansiedade.

Estudo realizado na cidade de Recife, Estado de Pernambuco, com residentes médicos e residentes não médicos aponta importante diferença na prevalência de transtornos mentais comuns entre os profissionais. Para os autores, essas diferenças podem ser justificadas pelas particularidades de cada profissão e, embora todos sejam profissionais de saúde, é comum se depararem com situações específicas que exigem atitudes e responsabilidades em níveis diferentes, podem gerar maior ou menor desgaste ao profissional (Carvalho et al., 2013). Em relação à depressão, os resultados mostram percentuais importantes de profissionais com algum nível da doença, especialmente nas áreas de Nutrição, Farmácia, Enfermagem, Psicologia, Serviço Social e Terapia Ocupacional. Resultados semelhantes foram encontrados em estudos com residentes médicos de radiologia - 56,0\% (Silva et al., 2010) e estudantes de enfermagem - 28,6\% para licenciatura e $15,4 \%$ para bacharelado (Furegato, Santos \& Silva, 2010).

Destaca-se, ainda, que estudos sobre Síndrome de Burnout com médicos residentes em Goiânia, Estado de Goiás (Soares et al., 2012) e na cidade de São Paulo, Estado de São Paulo (Fabichak, Silva-Junior \& Morrone, 2014), e residentes multiprofissionais no Estado do Rio Grande do Sul (Guido et al., 2012), relatam que os profissionais apresentam altos índices de exaustão emocional $(50,0 \%, 75,0 \%$ e $37,0 \%$, respetivamente), com risco para Síndrome de Burnout de 23,61\%, 50,0\% e 27,0\%, na devida ordem. 
Quando analisamos a associação entre ansiedade e depressão entre os profissionais estudados, os resultados encontrados (19,5\% dos profissionais com ansiedade e depressão associadas) confirmam a magnitude destes transtornos nos profissionais estudados, pertencentes à população de risco para transtornos emocionais e comportamentais (Silva et al., 2010).

Ao comparar profissionais de programas de aprimoramento e aperfeiçoamento profissional, com aqueles inseridos em programas de residência médica e/ou residência multiprofissional, destaca-se que o ambiente e o processo de trabalho são os mesmos para ambos os profissionais, havendo apenas distinção da carga horária total a ser cumprida que, para os aprimorandos e aperfeiçoandos é de 40 horas semanais (FAMERP, 2015; Secretaria de Estado da Saúde, 2015) e para os residentes médicos e multiprofissionais é de 60 horas semanais (Lei $n^{\circ} 12.514$; Portaria Interministerial $n^{\circ} 1.077$ ). Acerca desta questão, sabe-se que, se o número de horas trabalhadas for menor, o nível de exaustão emocional e despersonalização diminui (Asaiag, Perotta, Martins \& Tempski, 2010).

Entretanto, fatores comuns a todas as categorias profissionais inseridas em programas de formação são descritos como desgastantes, como a relação com pacientes rebeldes, a comunicação de uma doença grave, o medo de contrair um doença transmissível, o lidar com a morte, a rotatividade entre diferentes setores, o medo de cometer erros, a falta de supervisão adequada, a falta de infraestrutura para atendimento, sobrecarga de trabalho, entre outros (Carvalho et al., 2013; Franco et al., 2011; Silva et al., 2010).

A associação desses fatores comportamentais e ambientais, as condições de trabalho do local de estudo e as características sociodemográficas dos profissionais estudados, pode justificar os percentuais de profissionais com depressão entre aqueles que referiram não estar satisfeitos com o programa $(42,0 \%)$ e os que já pensaram em desistir (36,8\%).

Finalmente, para alguns pesquisadores, ações como supervisão constante e permanente dos profissionais em treinamento; melhor organização do trabalho, com equilíbrio entre a realização das atividades profissionais e o descanso; ações de promoção de saúde e bem-estar; reconhecimento e valorização institucional são importantes e contribuem para reduzir o estresse, evitando desgastes e o desenvolvimento de ansiedade e depressão (Rotta et al., 2016; Fabichak et al., 2014; Soares et al., 2012; Silva et al., 2010).

\section{CONCLUSÃO}

Este estudo permitiu identificar o perfil de susceptibilidade para desgastes físicos e emocionais relacionados ao trabalho.

Os índices de ansiedade e depressão encontrados nos profissionais estudados são significativos e evidenciam a presença de fatores desestimulantes e/ou desgastantes relacionados ao processo de formação nos programas avaliados.

Os resultados evidenciam a necessidade de ações de promoção de saúde e bem-estar dos trabalhadores. A implementação de medidas que identifiquem precocemente sintomas de ansiedade e estresse e promovam controle das causas de ansiedade e estresse; previna incapacidades individuais decorrentes destes transtornos. Este estudo demonstrou que transtornos emocionais, conhecidamente frequentes entre residentes médicos, também acometem os profissionais dos programas de aprimoramento e aperfeiçoamento. Contudo, o delineamento transversal deste estudo representa uma limitação por não permitir o estabelecimento de relações causais, o que requer a realização de novos estudos, para a investigação da relação de causa e efeito entre os transtornos avaliados, as características comportamentais, ambientais e as condições de trabalho desses profissionais.

A população restrita a uma única instituição impossibilita estender os resultados para o universo dos profissionais inseridos em programas de aprimoramento e aperfeiçoamento profissional, apontando a necessidade de investigações com profissionais de diferentes instituições/localidades.

Assim, conhecer a realidade dos profissionais matriculados em programas de aprimoramento e aperfeiçoamento profissional em saúde é fundamental para a análise e intervenção sobre os fatores negativos, bem como fortalecimento dos aspectos positivos do ambiente/processo de formação, assegurando maior desenvolvimento do profissional e melhoria na qualidade da assistência aos utentes dos serviços de saúde.

\section{REFERÊNCIAS BIBLIOGRÁFICAS}

Apóstolo, J. L. A., Figueiredo, M. H., Mendes, A. C., \& Rodrigues, M. A. (2011). Depression, anxiety and stress in primary health care users. Revista Latino-Americana de Enfermagem, 19(2), 348-353. doi: 10.1590/S010411692011000200017 
Asaiag, P. E., Perotta, B., Martins, M. A., \& Tempski, P. (2010). Avaliação da qualidade de vida, sonolência diurna e burnout em médicos residentes. Revista Brasileira de Educação Médica, 34(3), 422-429. doi: 10.1590/ S0100-55022010000300012

Carvalho, C. N., Melo-Filho, D. A., Carvalho, J. A. G., \& Amorim, A. C. G. (2013). Prevalência e fatores associados aos transtornos mentais comuns em residentes médicos e da área multiprofissional. Jornal Brasileiro de Psiquiatria, 62(1), 38-45. doi: 10.1590/S004720852013000100006

Cunha, J. A. (2001). Manual da versão em português das Escalas Beck. São Paulo: Casa do Psicólogo Livraria e Editora.

Fabichak, C., Silva-Junior, J. S., \& Morrone, L. C. (2014). Síndrome de burnout em médicos residentes e preditores organizacionais do trabalho. Revista Brasileira de Medicina do Trabalho, 12(2), 79-84.

Faculdade de Medicina de São José do Rio Preto, Comissão de Aprimoramento e Aperfeiçoamento da FUNFARME e FAMERP. (2015). Processo Seletivo 2016. São José do Rio Preto: Faculdade de Medicina de São José do Rio Preto.

Fernandes, L. F. B., Miyazaki, M. C. O. S., \& Silvares, E. F. M. (2015). Caracterização da supervisão em um centro formador de Psicologia da Saúde. Estudos de Psicologia (Campinas), 32(3), 499-509. doi: 10.1590/0103166X2015000300014

Franco, G. P., Barros, A. L. B. L., Nogueira-Martins, L. A., \& Zeitoun, S. S. (2011). Burnout em residentes de enfermagem. Revista da Escola de Enfermagem da USP, 45(1), 12-18. doi: 10.1590/S0080-62342011000100002

Furegato, A. R. F., Santos, J. L. F., \& Silva, E. C. (2010). Depressão entre estudantes de dois cursos de enfermagem: Autoavaliação da saúde e fatores associados. Revista Brasileira de Enfermagem, 63(4), 509-516. doi: 10.1590/S0034-71672010000400002

Gomes, R. K., \& Oliveira, V. B. D. (2013). Depressão, ansiedade e suporte social em profissionais de enfermagem. Boletim de Psicologia, 63(138), 23-33.
Guido, L. A., Silva, R. M., Goulart, C. T., Bolzan, M. E. O., \& Lopes, L. F. D. (2012). Síndrome de Burnout em residentes multiprofissionais de uma universidade pública. Revista da Escola de Enfermagem da USP, 46(6), 14771483. doi: 10.1590/S0080-62342012000600027

Hospital de Base de São José do Rio Preto. (2016). O hospital. Recuperado em 26 janeiro, 2016, de http:// www.hospitaldebase.com.br/hospital-de-base

Lei $\mathrm{n}^{\circ}$ 12.514, de 28 de outubro de 2011. Acedido em http://www.planalto.gov.br/ccivil_03/_Ato20112014/2011/Lei/L12514.htm\#art1

Lourenção, L. G., Moscardini, A. C., \& Soler, Z. A. S. G. (2013). Quality of life of non-medical resident professionals. Journal of Nursing UFPE on line, 7(11), 63366345. doi: 10.5205/reuol.3794-32322-1-ED.0711201304

Portaria Interministerial $\mathrm{n}^{\circ} 1.077$, de 12 de novembro de 2009.

Rotta, D. S., Pinto, M. H., Lourenção, L. G., Teixeira, P. R., Gonsalez, E. G., \& Gazetta, C. E. (2016). Níveis de ansiedade e depressão entre residentes multiprofissionais em saúde. Revista Rene, 17(3), 372-377.

Secretaria de Estado da Saúde. Programa de Aprimoramento Profissional. (2015). Manual de orientações técnicas e administrativas. São Paulo: Fundação do Desenvolvimento Administrativo.

Schmidt, D. R. C., Dantas, R. A. S., \& Marziale, M. H. P. (2011). Ansiedade e depressão entre profissionais de enfermagem que atuam em blocos cirúrgicos. Revista da Escola de Enfermagem da USP, 45(2), 487-493.

Silva, G. C. C., Koch, H. A., Sousa, E. G., Gasparetto, E., \& Buys, R. C. (2010). Ansiedade e depressão em residentes em Radiologia e Diagnóstico por Imagem. Revista Brasileira de Educação Médica, 34(2), 199-206.

Soares, L. R., Lopes, T. M. O., Silva, M. A. O., Ribeiro, M. V. A., Almeida Júnior, M. P., Silva, R. A., ... \& Chen Chen, L. (2012). Burnout e pensamentos suicidas em médicos residentes de hospital universitário. Revista Brasileira de Educação Médica, 36(1), 77-82.

World Health Organization. (2015). Mental disorders. Acedido em http://www.who.int/mediacentre/factsheets/fs396/en/ 